\title{
Curcumin pyrazole blocks lipopolysaccharide-induced inflammation via suppression of JNK activation in RAW 264.7 macrophages
}

Nathnarin Somchit, ${ }^{1}$ Rungruedee Kimseng, ${ }^{1}$ Rana Dhar, ${ }^{1}$ Poonsit Hiransai, ${ }^{1}$ Chatchawan Changtam,${ }^{2}$ Apichart Suksamrarn, ${ }^{3}$ Wilanee Chunglok, ${ }^{4}$ Warangkana Chunglok ${ }^{1}$

\begin{abstract}
Background: Targeting inflammatory macrophages and their products is an effective method for controlling inflammation. The pyrazole analog of curcumin (curcumin pyrazole, PYR) has been reported to possess superior anti-inflammatory activity to curcumin (CUR). However, the role of PYR anti-inflammatory activity in macrophages has not yet been elucidated.

Objective: To examine the anti-inflammatory effects of PYR and CUR in lipopolysaccharide (LPS)-activated RAW 264.7 macrophages and determine the role of mitogen-activated protein kinases (MAPK) in their activity.

Methods: Nitrite level was investigated by the Griess assay. The expression of inducible nitric oxide (NO) synthase, cyclooxygenase-2 (COX-2), and MAPK proteins were analyzed by western blot analysis. The pro-inflammatory cytokines tumor necrosis factor (TNF)- $\alpha$, interleukin (IL)-1 $\beta$, and interleukin-6 (IL-6) were measured by enzyme-linked immunosorbent assay.

Results: LPS-induced NO secretion in RAW 264.7 macrophages was potently inhibited by PYR $\left(\mathrm{IC}_{50}=3.7 \pm 0.16 \mu \mathrm{M}\right)$, at a higher efficacy than CUR $\left(\mathrm{IC}_{50}=11.0 \pm 0.59 \mu \mathrm{M}\right)$. Treatment with identical concentrations of PYR and CUR demonstrated that PYR drastically inhibited iNOS and COX-2 expression, whereas CUR only blocked COX-2. PYR reduced the LPS-induced secretion of TNF- $\alpha$ to a greater extent than CUR and both similarly reduced IL- $1 \beta$ and IL- 6 levels. Activation of c-Jun N-terminal kinase (JNK) MAPK was significantly decreased in LPS-activated RAW 264.7 macrophages upon PYR but not CUR treatment.
\end{abstract}

Conclusion: PYR exhibited a more potent anti-inflammatory activity than CUR. This activity is partly mediated by PYR-depended inhibition of the JNK signaling pathway and underscores the utility of PYR as an anti-inflammatory agent in macrophages.

Keywords: curcumin, pyrazole, iNOS, COX-2, MAPK

\section{From:}

${ }^{1}$ Molecular Medicine and Cancer Biology Research Unit, School of Allied Health Sciences, Walailak University, Nakhon Si Thammarat, Thailand

${ }^{2}$ Faculty of Science and Technology, Huachiew Chalermprakiet University, Samutprakarn, Thailand

Department of Chemistry and Center of Excellence for Innovation in Chemistry, Faculty of Science, Ramkhamhaeng University, Bangkok, Thailand

${ }^{4}$ Department of Microbiology, Faculty of Science, Prince of Songkla University, Thailand

\section{Corresponding author:}

Warangkana Chunglok

Molecular Medicine and Cancer Biology Research Unit, School of Allied

Health Sciences, Walailak University, Nakhon Si Thammarat, 80161,

Thailand

Email: cwarang@wu.ac.th, aonmu@hotmail.com

\section{Introduction}

Macrophages are key mediators of the pathophysiological component of inflammation. Overproduction of reactive oxygen/nitrogen species and pro-inflammatory cytokines by macrophages recruits additional immune cells that contribute to severe inflammatory reactions and tissue destruction. ${ }^{1}$ 
Nonsteroidal anti-inflammatory drugs are used extensively in the management of inflammatory conditions; however, their use is associated with drug-related morbidity representing significant adverse gastrointestinal, renal, and cardiovascular effects. ${ }^{2,3}$ As such, there is growing interest in the development of more efficient anti-inflammatory drugs derived from natural sources that induce fewer side effects.

Several natural products derived from medicinal plants from Thailand display anti-inflammatory properties. ${ }^{4}$ Among them, Curcuma longa L. rhizomes have been investigated as a source of compounds with anti-oxidative, anti-cancer, and anti-inflammatory activities in both in vitro and in vivo studies. ${ }^{5,6,7}$ Curcuminoids are responsible for these activities, the principle compound among them being curcumin (CUR). As such, CUR is a good starting compound for the design of new drugs. Indeed, many stable analogs have been synthesized and investigated. Among these, curcumin pyrazole (PYR), a pyrazole analog, has been found to possess significantly enhanced cycloox genase (COX)-2/ COX-1 selectivity and anti-inflammatory activity in a carrageenan-induced paw edema rat model versus CUR. ${ }^{8}$ PYR and a prenylated pyrazole analog were found to be potent and selective inhibitors of 5-lipoxygenase, a key enzyme implicated in inflammation-associated induction of cancer. ${ }^{9}$ Another CUR-based pyrazole analogue, CNB-001, significantly suppressed interleukin (IL)-6, tumor necrosis factor (TNF)- $\alpha$, and serine peptidase inhibitor, clade E member 1 production both in an inflamed human bronchial epithelial cell model and an in vivo murine model of asthma. ${ }^{10}$

Pattern recognition receptors such as toll-like receptors, RIG-I-like receptors, NOD-like receptors, and C-type lectin receptors activate both mitogen-activated protein kinases (MAPKs) and nuclear factor (NF)- $\kappa B$ (NF- $\kappa B$ ) pathways, which are responsible for stimulation of inflammatory immune responses in macrophages. ${ }^{11}$ As such, MAPKs represent an attractive therapeutic target for the prevention of excessive inflammation and treatment of inflammatory diseases. Three distinct MAPK pathways have been extensively studied, including extracellular signal-regulated kinase (ERK), p38 MAPK, and c-Jun N-terminal kinase (JNK). CUR has been shown to block inducible nitric oxide synthase (iNOS) tyrosine phosphorylation through the inhibition of ERK1/212 and suppress pro-inflammatory cytokine production by inhibiting p38 MAPK $^{13}$ and JNK in activated RAW 264.7 macrophages. ${ }^{14}$ However, whether PYR also acts through inhibition of MAPK is unknown. In the present study, we investigated the anti-inflammatory activities of both CUR and PYR in lipopolysaccharide (LPS)-activated RAW 264.7 macrophages with specific emphasis on their underlying mechanisms of action against MAPKs.

\section{Methods \\ Materials}

CUR was obtained from C. longa L. rhizomes, and PYR was synthesized as previously described. ${ }^{15}$ Both compounds were dissolved in dimethyl sulfoxide (DMSO) and diluted with complete media to obtain final concentrations of 2-64 $\mu \mathrm{M}$. DMSO was used as a vehicle control at a concentration of $0.5 \%$, which is comparable to that of $16 \mu \mathrm{M}$ of the compounds used in the experimental conditions, and was not toxic to the cells.

\section{Cell Culture}

The murine macrophage cell line RAW 264.7 (ATCC, Manassas, VA) was seeded at a density of $3.0 \times 10^{5}$ cells $/ \mathrm{cm}^{2}$ in RPMI-1640 (PAA Laboratories GmbH, Pasching, Austria) supplemented with $10 \%$ fetal bovine serum (Biochrom $\mathrm{GmbH}$, Berlin, Germany), 1\% penicillin/streptomycin (PAA Laboratories $\mathrm{GmbH}$, Pasching, Austria), and $2 \mathrm{mM}$ stable glutamine (Life Technologies, Carlsbad, California). Cells were grown at $37^{\circ} \mathrm{C}$ in a humidified $5 \% \mathrm{CO}_{2}$ atmosphere under standard conditions.

\section{Measurement of cytotoxicity}

RAW 264.7 macrophages at a density of $3.0 \times 10^{5}$ cells/ $\mathrm{cm}^{2}$ were cultured for $24 \mathrm{~h}$ at which time they were treated with CUR and PYR at various concentrations ranging from 0 to $64 \mu \mathrm{M}$. DMSO was used as vehicle control. The treated cells were incubated for an additional $24 \mathrm{~h}$ and the spent medium was then removed and $200 \mu \mathrm{l}$ of $0.5 \mathrm{mg} / \mathrm{ml}$ of 3-(4,5-dimethylthiazolyl-2)-2,5-diphenyltetrazolium bromide (MTT) (Sigma-Aldrich, St. Louis, MO) solution was added into each well and incubated at $37^{\circ} \mathrm{C}$ in a $\mathrm{CO}_{2}$ incubator for $3 \mathrm{~h}$. The resulting formazan crystals were dissolved in DMSO, and the absorbance for each well was detected at $560 \mathrm{~nm}$ using a microplate reader. Data were obtained from three independent experiments in triplicate. Cell viability was calculated using the following equation:

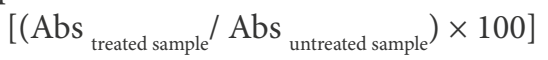

\section{Measurement of nitric oxide (NO) production}

RAW 264.7 macrophages at a density of $3.0 \times 10^{5}$ cells/ $\mathrm{cm}^{2}$ were treated with CUR, PYR, dexamethasone (DEX) or DMSO (vehicle control) in the presence of LPS (5 ng/ml from Escherichia coli serotype O111:B4, Sigma-Aldrich) for $24 \mathrm{~h}$. In another experiment, RAW 264.7 cells were pretreated with 50 $\mu \mathrm{M}$ U0126 (MEK 1/2 inhibitor), $50 \mu \mathrm{M}$ SB203580 (p38 MAPK inhibitor), and $50 \mu \mathrm{M}$ SP600125 (JNK inhibitor; all from Sigma-Aldrich) for $30 \mathrm{~min}$. After removing the inhibitors, cells were incubated with LPS for $24 \mathrm{~h}$. The conditioned culture media was collected and centrifuged at $1620 \times \mathrm{g}$ in a refrigerated centrifuge at $4^{\circ} \mathrm{C}$ for $10 \mathrm{~min}$. The supernatant was measured for nitrite levels by the Griess assay. ${ }^{16}$ Optical density was measured at $548 \mathrm{~nm}$ and the nitrite level was determined in $\mu \mathrm{M}$ from a nitrite standard curve $\left(r^{2}>0.990\right)$. Data were expressed as the percentage of nitrite levels relative to the nitrite obtained from DMSO control in LPS-treated cells.

\section{Selectivity index}

Selectivity index (SI) value was calculated in order to determine the selectivity of CUR and PYR on NO inhibition. SI was obtained by dividing the half maximal cytotoxic concentration $\left(\mathrm{CC}_{50}\right)$ value for cytotoxicity by the half maximal inhibitory concentration $\left(\mathrm{IC}_{50}\right)$ value for NO inhibition. ${ }^{17}$

\section{Measurement of inflammatory cytokines}

RAW 264.7 macrophages at a density of $3.0 \times 10^{5}$ cells/ $\mathrm{cm}^{2}$ were treated with CUR, PYR, or DMSO (vehicle control) 
in the presence of LPS (5 ng/ml) for $24 \mathrm{~h}$. Culture media was collected and centrifuged at $1620 \times \mathrm{g}$ in a refrigerated centrifuge at $4^{\circ} \mathrm{C}$ for $10 \mathrm{~min}$ and examined for the presence of mouse TNF- $\alpha$, IL-6 (Millipore, Billerica, MA), and IL-1 $\beta$ (Abcam, Cambridge, United Kingdom) by enzyme-linked immunosorbent assay (ELISA) according to the manufacturers' specifications. Inflammatory cytokine levels were determined against standard curves $\left(r^{2}>0.990\right)$

\section{Western blot analysis}

RAW 264.7 macrophages at a density of $3.0 \times 10^{5} \mathrm{cells} / \mathrm{cm}^{2}$ were treated with CUR, PYR, or DMSO (vehicle control) for $30 \mathrm{~min}$, followed by LPS ( $5 \mathrm{ng} / \mathrm{ml})$ treatment for $20 \mathrm{~min}$. Cells were harvested, washed three times with ice-cold phosphate buffered saline and lysed by sonication in sonication buffer containing protease inhibitors. Cell lysates was centrifuged at $4^{\circ} \mathrm{C}$ for $10 \mathrm{~min}$. Supernatants were removed and protein concentrations were measured by Bradford assay. iNOS, COX-2, phospho-JNK, total JNK, phospho-p38, total p38, phospho-ERK1/2, and total ERK1/2 protein expression in cell lysates were then determined by western blot analysis. Briefly, $50 \mu \mathrm{g}$ protein of each sample was loaded onto $10 \%$ sodium dodecyl sulfate-polyacrylamide gel and transferred to polyvinylidene fluoride membranes. The membranes were incubated with anti-iNOS (1:1000), anti-COX-2 (1:1000), anti-phospho-JNK (1:500), anti-JNK (1:1000), anti-phospho -p38 (1:500), anti-p38 (1:1000), anti-phospho-ERK1/2 (1:1000), and anti-ERK1/2 (1:1000) antibodies in 5\% BSA for overnight at $4^{\circ} \mathrm{C}$. All antibodies were purchased from Cell Signaling Technology (Danvers, MA). Membranes were then washed three times with TBST, incubated in anti-rabbit secondary antibody (1:5000) for $1 \mathrm{~h}$ at room temperature, washed again and detected with a chemiluminescent system using Hyperfilm and ECL plus (GE Healthcare, Buckinghamshire, UK). For the internal loading control, membranes were stripped and reprobed with anti $\beta$-actin antibody (1:2000).

\section{Statistical Analysis}

Results are expressed as the mean \pm SEM of three independent experiments. The differences of data were analyzed using ANOVA or independent $t$-test. Significant difference was considered at $p<0.05$. Statistical analysis was performed with SPSS (version 17.0).

\section{Results}

\section{Effects of CUR and PYR on cytotoxicity and NO production}

Treatment of RAW 264.7 macrophages with CUR and PYR (Figure 1A,B) at concentrations up to $8 \mu \mathrm{M}$ did not affect cell growth or viability (Figure 2A). However, CUR and PYR were found to have cytotoxic effects with $\mathrm{CC}_{50}$ values of $27.0 \pm 0.54$ and $24.0 \pm 0.73 \mu \mathrm{M}$, respectively (Figure 2C). PYR concentrations of 2,4 , and $8 \mu \mathrm{M}$ significantly inhibited LPS-induced macrophage secretion of NO in a dose-dependent manner $(p<0.05)$ (Figure $2 \mathrm{~B})$, with an $\mathrm{IC}_{50}$ of $3.70 \pm 0.16 \mu \mathrm{M}$, whereas CUR exhibited an $\mathrm{IC}_{50}$ of $11.0 \pm 0.59 \mu \mathrm{M}$ (Figure 2C). As a positive control, DEX inhibited NO secretion with an $\mathrm{IC}_{50}$ of $5.5 \pm 0.33 \mu \mathrm{M}$. To assess the selectivity of NO inhibition, SI values were calculated. CUR and PYR exhibited SI values of 2.5
A<smiles>COc1cc(/C=C/C(=O)CC(=O)/C=C/c2ccc(O)c(OC)c2)ccc1O</smiles>

B<smiles>COc1cc(/C=C/c2cc(/C=C/c3ccc(O)c(OC)c3)[nH]n2)ccc1O</smiles>

Figure 1. Chemical structures of (A) CUR and (B) PYR.

A
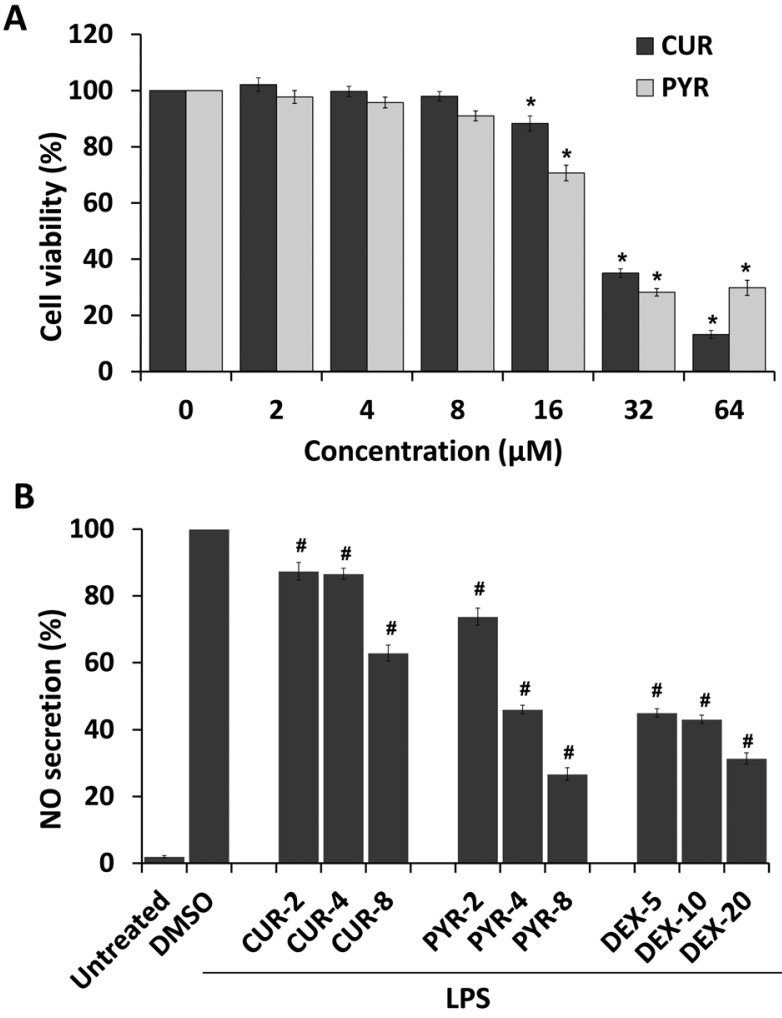

C

\begin{tabular}{|c|c|c|c|}
\hline Compounds & $\mathrm{CC}_{50}(\mu \mathrm{M})$ & $\mathrm{IC}_{50}(\mu \mathrm{M})$ & $\mathrm{SI}$ \\
\hline CUR & $27.0 \pm 0.54$ & $11.0 \pm 0.59$ & 2.5 \\
\hline PYR & $24.0 \pm 0.73$ & $3.70 \pm 0.16$ & 6.5 \\
\hline
\end{tabular}

Figure 2. Cell viability and NO inhibitory activity.

(A) To assess viability, RAW 264.7 cells were treated with 2-64 $\mu \mathrm{M}$ of CUR or PYR for $24 \mathrm{~h}$ and an MTT assay was performed. (B) To assess nitric oxide production, RAW 264.7 cells were treated with $2,4,8$, and $16 \mu \mathrm{M}$ of the CUR, PYR, or DEX for $24 \mathrm{~h}$ in the presence or absence of $5 \mathrm{ng} / \mathrm{ml} \mathrm{LPS}$. Culture supernatant was then subjected to the Griess assay. (C) $\mathrm{CC}_{50}$ and $\mathrm{IC}_{50}$ values are expressed as the mean \pm SEM of three-independent experiments. ${ }^{*} p<0.05$; significantly different from $0 \mu \mathrm{M}$ (DMSO control). ${ }^{*} p<0.05$; significantly different from LPS-activated RAW 264.7 macrophages treated with DMSO. 
and 6.5, respectively (Figure 2C). The SI value obtained from PYR represents high selectivity for inhibition of NO secretion rather than general toxicity induction.

\section{Effect of PYR on iNOS and COX-2 protein expression}

To evaluate whether the inhibition of NO secretion was associated with the reduction of iNOS expression, western blot analysis of iNOS protein was performed. PYR treatment was found to significantly decrease iNOS protein expression in LPS-activated macrophages compared with the DMSO-treated control cells $(p<0.05)$ (Figure 3A). We found that PYR, CUR, and DEX all dramatically down-regulated the expression of COX-2, another key inflammatory protein, in LPS-activated macrophages treated with DMSO $(p<0.05)$ (Figure 3B). Therefore, PYR has the potential to suppress both iNOS and COX-2 protein expression.

\section{Effect of PYR on pro-inflammatory cytokine secretion}

To determine whether this inhibition of inflammatory signaling corresponded to reduced pro-inflammatory cytokine secretion, we investigated cytokine secretion in the LPS-activated macrophages by ELISA. PYR dramatically
A

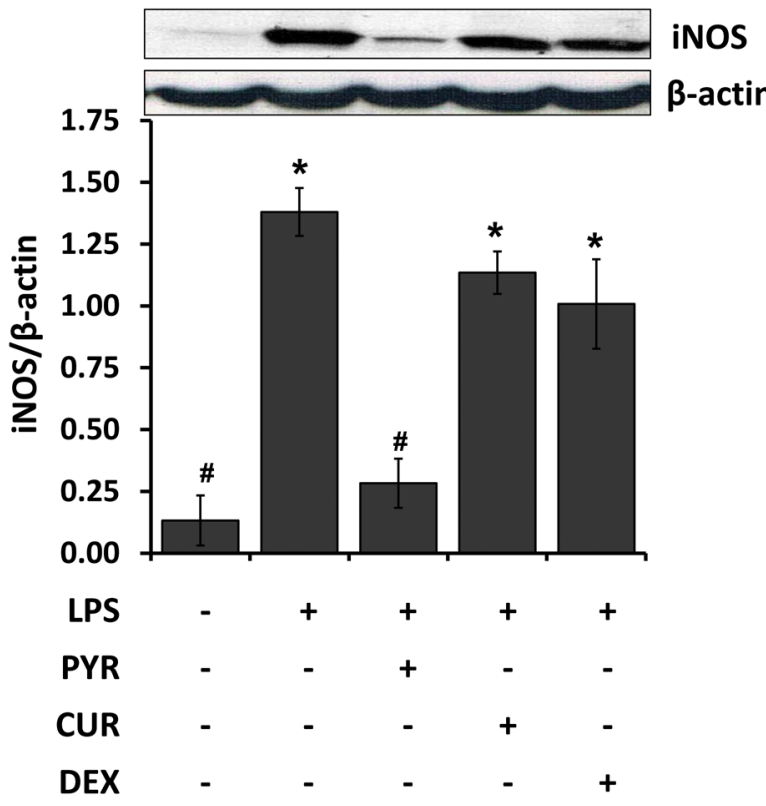

B
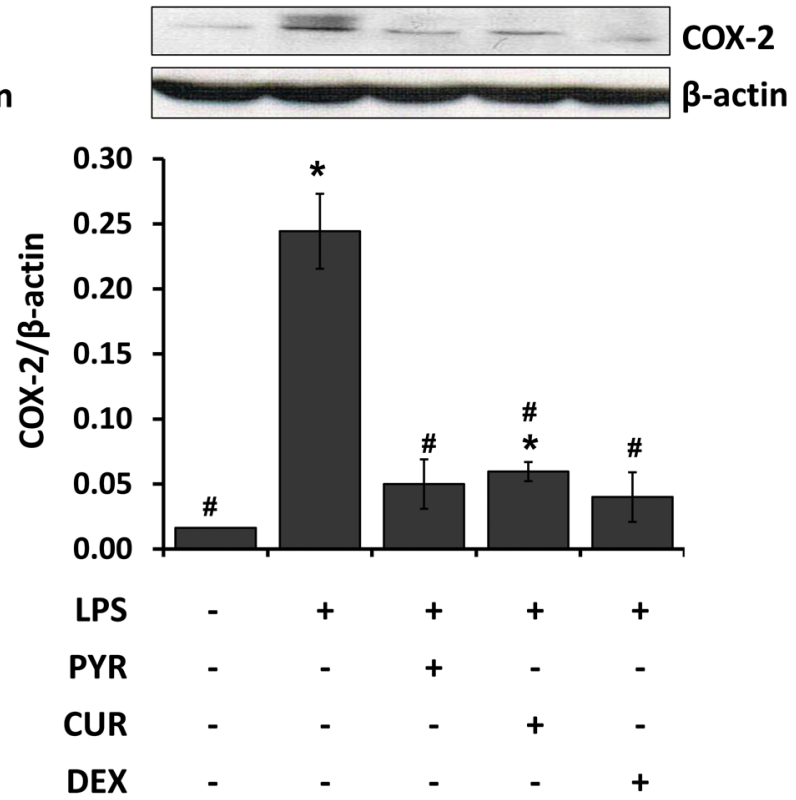

Figure 3. Effects of CUR and PYR on the expression of iNOS and COX-2.

RAW 264.7 cells were treated with $8 \mu \mathrm{M}$ of the CUR or PYR for $24 \mathrm{~h}$ in the presence or absence of $5 \mathrm{ng} / \mathrm{ml}$ of LPS and an western blot analysis of the (A) iNOS and (B) COX-2 proteins was performed. The results shown are representative of three independent experiments. Values are expressed as mean \pm SEM. ${ }^{\star} p<0.05$ : significantly different from the untreated control. ${ }^{\#} p<0.05$ : significantly different from LPS-activated RAW 264.7 macrophages treated with DMSO.
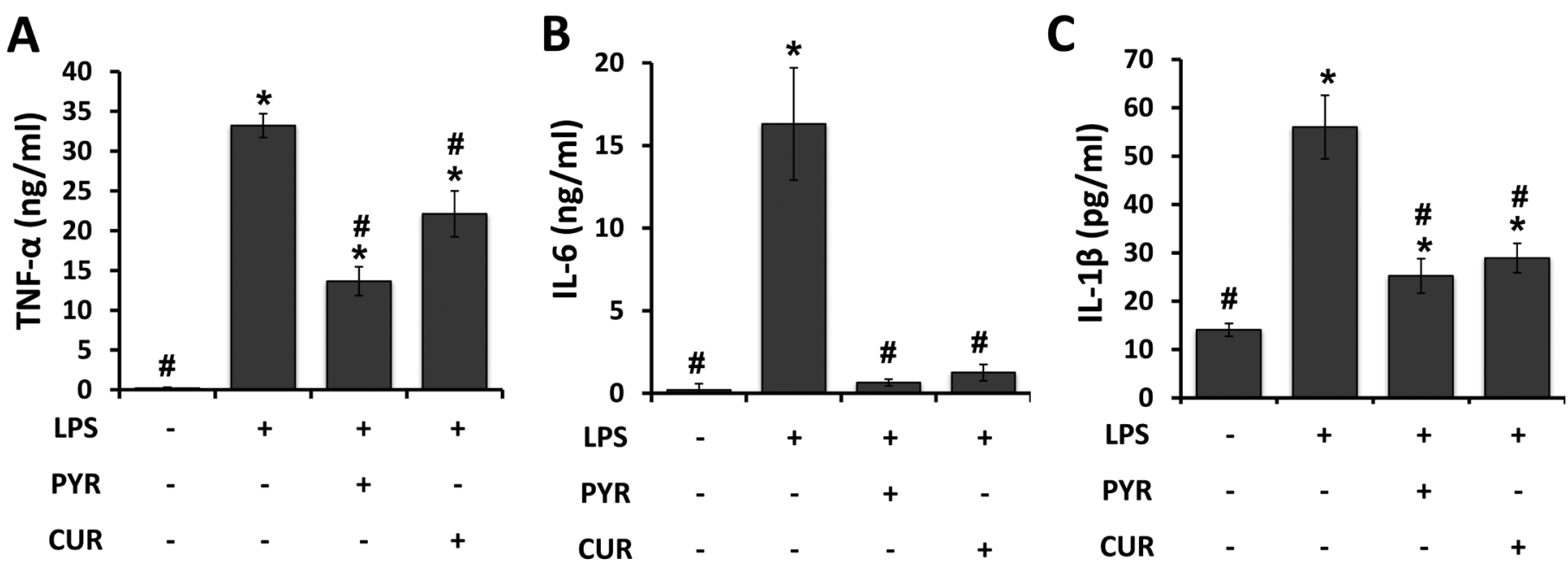

Figure 4. Effects of CUR and PYR on the levels of pro-inflammatory cytokines.

RAW 264.7 cells were treated with $8 \mu \mathrm{M}$ of the CUR or PYR for $24 \mathrm{~h}$ in the presence or absence of $5 \mathrm{ng} / \mathrm{ml}$ of LPS. Cultured supernatant was subjected to ELISA for (A) TNF- $\alpha$, (B) IL-6, and (C) IL-1 $\beta$. The results shown were obtained from three independent experiments. Values are expressed as means \pm SEM. ${ }^{\star} p<0.05$ : significantly different from the untreated control. ${ }^{\#} p<0.05$ : significantly different from LPS-activated RAW 264.7 macrophages treated with DMSO. 
decreased the expression of the pro-inflammatory cytokines TNF- $\alpha$ (Figure 4A), IL-6 (Figure 4B), and IL-1 $\beta$ (Figure 4C) by $59 \%, 96 \%$, and $55 \%$, respectively. The anti-inflammatory action of PYR on the inhibition of TNF-a production was greater than that of CUR by $26 \%$. Furthermore, suppression of iNOS/NO and COX-2 was concomitant with the inhibition of pro-inflammatory cytokines by PYR.

\section{Effect of PYR on the MAPKs phosphorylation}

To determine whether PYR affected signaling pathways of inflammation, we pretreated macrophages with PYR for 30 min followed by LPS treatment for $20 \mathrm{~min}$ and examined the expression and phosphorylation of MAPK proteins by western blot analysis. PYR induced a greater inhibition JNK activation than CUR in LPS-stimulated macrophages (Figure 5A,B). No further inhibition was observed for phospho-ERK $1 / 2$ and phospho-p38 MAPK. These results suggest that the JNK signaling pathway is involved in the enhanced activity of PYR in suppression of LPS-induced cytokine secretion in macrophages. To further investigate the role of the MAPK signaling pathway in LPS-induced NO secretion, the specific MAPK inhibitors MEK inhibitor U0126, p38 MAPK inhibitor SB203580, and JNK inhibitor SP600125 were used. All showed significant inhibition of NO secretion in LPS-activated RAW 264.7 macrophages in comparison with LPS alone (Figure 5C). These results indicate that MEK/ERK, p38 MAPK, and JNK contribute to LPS-induced NO production and that PYR may have anti-inflammatory benefit.
A

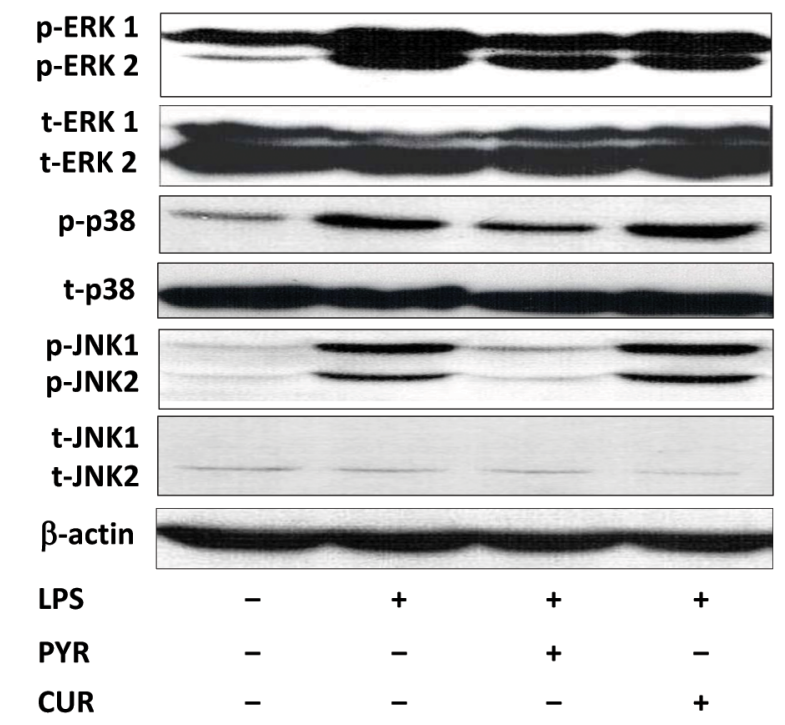

C

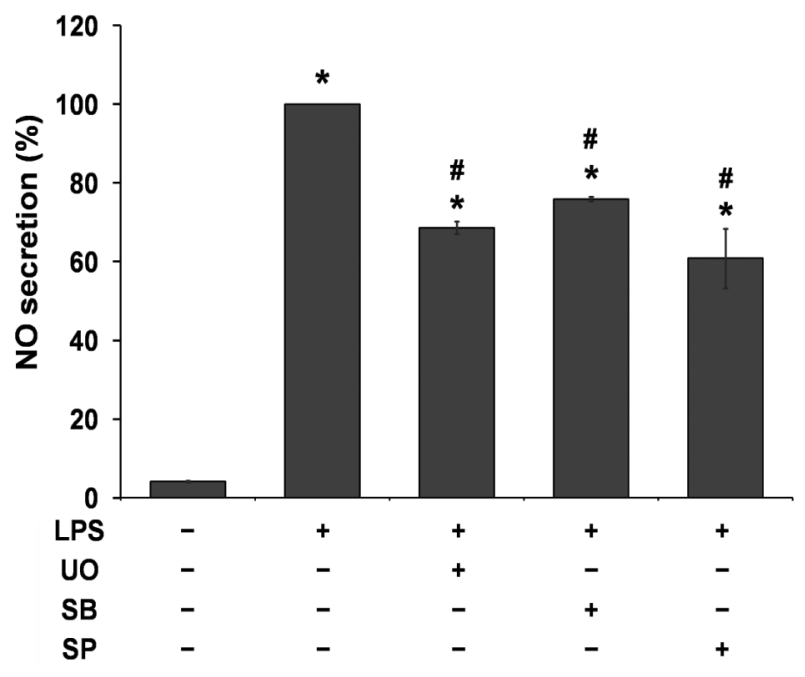

B
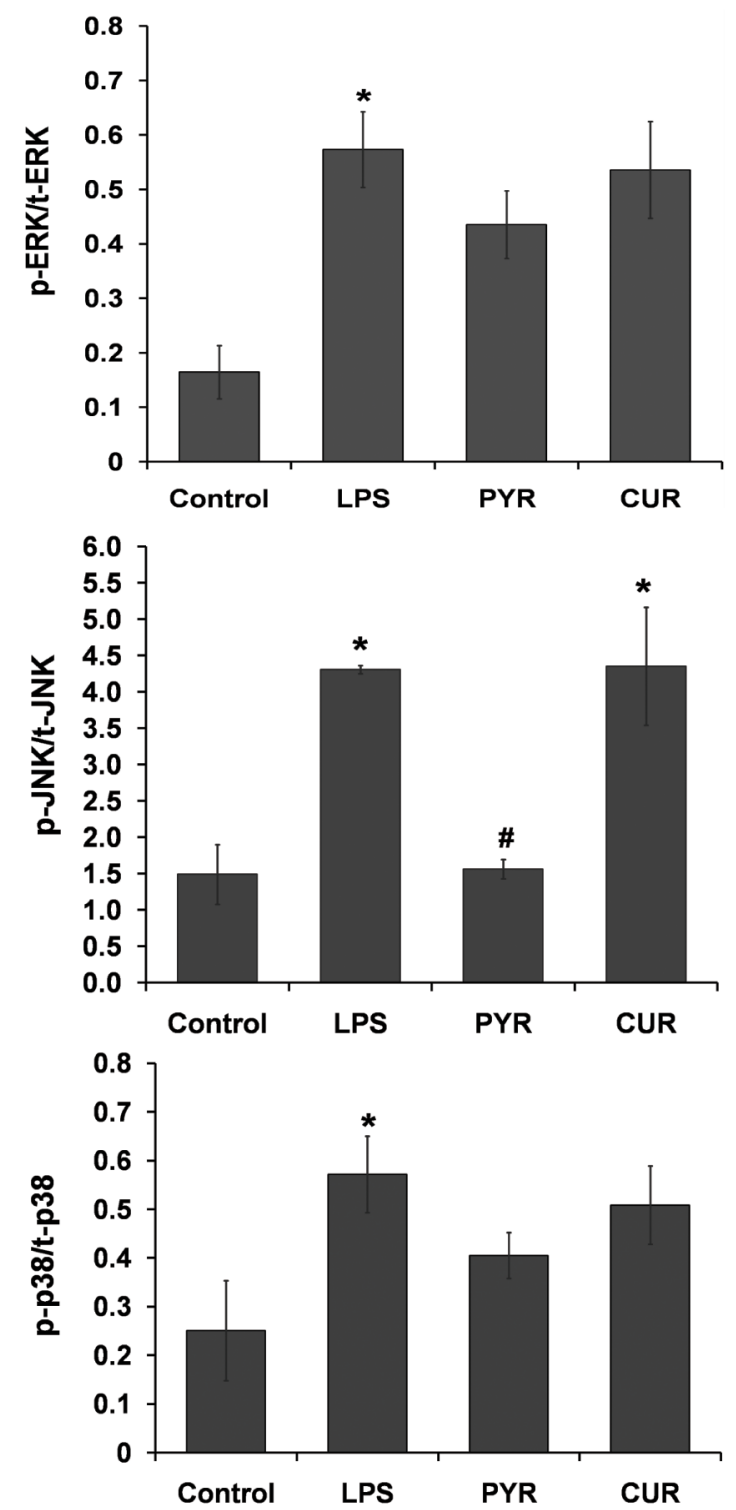

Figure 5. Effects of CUR and PYR on the expression of MAPK proteins. RAW 264.7 cells were pretreated with $8 \mu \mathrm{M}$ of the CUR or PYR for $30 \mathrm{~min}$ and thereafter treated with $5 \mathrm{ng} / \mathrm{ml}$ of LPS for $20 \mathrm{~min}$. (A) Western blot analysis of ERK, p38, and JNK was performed from three independent experiments. One representative blot is shown. (B) Relative expression levels of the phosphorylated forms of ERK, p38, and JNK proteins were determined by densitometric analysis. (C) RAW 264.7 cells were pretreated with $50 \mu \mathrm{M}$ of the MAPK inhibitors U0126, SB203580, and SP600125 for $30 \mathrm{~min}$ and by $5 \mathrm{ng} / \mathrm{mL}$ LPS for $24 \mathrm{~h}$ thereafter. Culture supernatant was then subjected to the Griess assay. Values are expressed as mean \pm SEM of three independent experiments in triplicate. ${ }^{*} p<0.05$ : significantly different from the untreated control. ${ }^{*} p<0.05$ : significantly different from LPS-activated RAW 264.7 macrophages treated with DMSO. 


\section{Discussion}

Macrophages are considered key targets for treating inflammatory diseases. In the present study, we found that PYR exhibited more potent anti-inflammatory activity than CUR in LPS-activated RAW 264.7 macrophages, particularly with regard to suppression of NO secretion, iNOS expression, and TNF- $\alpha$ secretion. The underlying mechanism of action is likely due in part to the selective blockade of JNK activation by PYR but not by CUR.

CUR is a potent anti-inflammatory compound isolated from $C$. longa L. $^{18}$ It was shown that curcuminoids at concentrations ranging from 1 to $10 \mu \mathrm{M}$ are not toxic to RAW 264.7 macrophages. ${ }^{13,19}$ However, CUR was previously found to induce cytotoxicity at higher concentrations $\left(\mathrm{CC}_{50}=28.8 \pm 0.80 \mu \mathrm{M}\right) \cdot{ }^{20}$ At a concentration of $12 \mu \mathrm{M}$, CUR significantly inhibits NO production by $50 \% .{ }^{21}$ CUR potently inhibits NO production with concomitant down-regulation of iNOS mRNA in LPS-activated microglial cells, ${ }^{22}$ promotes the ubiquitination and degradation of iNOS, suppresses iNOS activity after LPS stimulation, ${ }^{21}$ and was shown to scavenge NO directly, ${ }^{23}$ illustrating the multiple mechanisms through which CUR can inhibit inflammation. CUR also suppresses expression of the inflammatory mediator COX-2 in LPS-activated RAW 264.7 macrophages. ${ }^{24}$ In addition, CUR was shown to exert anti-inflammatory activity by binding directly to pro-inflammatory molecules (e.g., TNF- $\alpha$, COX-1, COX-2, and human a1-acid glycoprotein). ${ }^{25}$

Replacement of the $\beta$-diketo fragment of CUR by a pyrazole ring significantly enhanced its antioxidant and anti-inflammatory activities. ${ }^{89}$ A pyrazole derivative of curcumin, CNB-001, was shown to suppress IL-6, TNF- $\alpha$ and GM-CSF production in normal human bronchial epithelial cells and in a murine model of asthma. ${ }^{10}$ PYR has been shown to possess more potent activity than CUR in both inhibition of COX-2 in an in vitro, cell-free system and in rats using a carrageenan-induced paw edema assay. ${ }^{8}$ We hypothesized that PYR might also be used to inhibit LPS-induced inflammation in human macrophages. In agreement with previous analyses of CUR and PYR, we found PYR to be a superior anti-inflammatory agent.

Previously, the anti-inflammatory effects of CUR were demonstrated to be mediated by the inhibition of MAPK, ${ }^{13,26}$ nuclear factor NF- $\kappa B,{ }^{24,26}$ and signal transducer and activator of transcription 3 (STAT3) with concomitant activation of nuclear factor erythroid 2-related factor-2. ${ }^{9}$ MAPK plays a central role in the production of inflammatory mediators such as iNOS, COX-2, and inflammatory cytokines. CUR $(10-30 \mu \mathrm{M})$ has been shown to inhibit the phosphorylation of $\mathrm{ERK},{ }^{12} \mathrm{p} 38,{ }^{13}$ and $\mathrm{JNK}^{14}$ in activated mouse macrophages, as well as to suppress NF- $\kappa \mathrm{B}$ and toll-like receptor 4 signaling in activated macrophages derived from human monocytic THP-1 cells. ${ }^{26}$ Rat vascular smooth muscle cells pretreated with the specific ERK inhibitor PD98059 and p38 MAPK inhibitor SB203580, but not JNK inhibitor SP600125, stimulated with LPS, and treated with CUR exhibited reduced secretion of MCP-1, TNF- $\alpha$, and NO. ${ }^{27}$ Whereas in LPS-activated RAW 264.7 macrophages, each of the MAPK inhibitors U0126, SB203580, and SP600125 were shown to suppress TNF- $\alpha$ and IL-6 secretion and NO production. ${ }^{28,29}$ Thus, MAPK signaling pathways act as key mediators of inflammatory response that are targeted by CUR. In this study, our results have demonstrated that PYR exhibited a more potent anti-inflammatory activity than CUR and that this difference likely involves its suppression of JNK activation. Similarly, PYR induced a superior inhibition of the transcriptional activity of STAT3 in comparison to CUR and possessed the inhibition of NF- $\kappa \mathrm{B}$ transcriptional activity, which may affect the expression of genes involved in inflammation. ${ }^{9}$ Thus, PYR not only suppresses JNK activation in activated mouse macrophages but may also inhibit the NF- $\kappa B$ and STAT3 signaling pathways.

The present study showed that structural modification of CUR with a pyrazole ring to produce PYR, selectively increased NO inhibition and enhanced its anti-inflammatory activity. One possible explanation for this activity is PYR's improved stability in aqueous solutions at physiological $\mathrm{pH} .{ }^{9}$ CUR was found to be unstable and to rapidly degrade into trans-6-(4'-hydroxy-3'-methoxyphenyl)-2,4-dioxo-5-hexenal..$^{30}$ Additionally, PYR retains the hydrogen donor and acceptor properties of the enolized 1,3-dicarbonyl system of CUR, which is critical for the inhibition of inflammatory molecules such as 5-lipoxygenase in human neutrophils. ${ }^{9}$ Blockade of this key inflammatory enzyme by PYR may in turn affect other downstream inflammatory molecules, leading to a pronounced attenuation of inflammation.

\section{Conclusion}

In summary, we have demonstrated that PYR induces more anti-inflammatory effects and possesses enhanced anti-inflammatory activity over its parent compound, CUR, in macrophages. Taken as a whole, this study demonstrates that PYR potently inhibits JNK activation which is related to its anti-inflammatory activity. Further insights into the mechanisms underlying PYR's anti-inflammatory properties will be pursued in additional models of inflammation to facilitate development of PYR as a potential anti-inflammatory agent.

\section{Acknowledgments}

This research was funded in part by the Office of the Higher Education Commission MOE 0509 (7)/366.63, The Thailand Research Fund (DBG5980003), and the Center of Excellence for Innovation in Chemistry, Office of the Higher Education Commission. N. Somchit, R. Kimseng, and R. Dhar were supported by the Walailak University Fund for graduate studentships and the Thasala Hospital Radiology Department. We would like to thank Enago (https://www.enago.com/) for English language editing and reviewing of this manuscript.

\section{Conflict of interests}

The authors declare no conflicts of interest.

\section{References}

1. Lameijer MA, Tang J, Nahrendorf M, Beelen RH, Mulder WJ. Monocytes and macrophages as nanomedicinal targets for improved diagnosis and treatment of disease. Expert Rev Mol Diagn. 2013;13:567-80.

2. Bacchi S, Palumbo P, Sponta A, Coppolino MF. Clinical pharmacology of non-steroidal anti-inflammatory drugs: a review. Antiinflamm Antiallergy Agents Med Chem. 2012;11:52-64. 
3. Wehling M. Non-steroidal anti-inflammatory drug use in chronic pain conditions with special emphasis on the elderly and patients with relevant comorbidities: management and mitigation of risks and adverse effects. Eur J Clin Pharmacol. 2014;70:1159-72.

4. Ruangnoo S, Jaiaree N, Makchuchit S, Panthong S, Thongdeeying P, Itharat A. An in vitro inhibitory effect on RAW 264.7 cells by anti-inflammatory compounds from Smilax corbularia Kunth. Asian Pac J Allergy Immunol. 2012;30:268-74.

5. Guo LY, Cai XF, Lee JJ, Kang SS, Shin EM, Zhou HY, et al. Comparison of suppressive effects of demethoxycurcumin and bisdemethoxycurcumin on expressions of inflammatory mediators in vitro and in vivo. Arch Pharm Res. 2008;31:490-6.

6. Zhang L, Wu C, Zhao S, Yuan D, Lian G, Wang X, et al. Demethoxycurcumin, a natural derivative of curcumin attenuates LPS-induced pro-inflammatory responses through down-regulation of intracellular ROS-related MAPK/NF-kappaB signaling pathways in N9 microglia induced by lipopolysaccharide. Int Immunopharmacol. 2010;10:331-8.

7. Vallianou NG, Evangelopoulos A, Schizas N, Kazazis C. Potential anticancer properties and mechanisms of action of curcumin. Anticancer Res. 2015;35:645-51.

8. Selvam C, Jachak SM, Thilagavathi R, Chakraborti AK. Design, synthesis, biological evaluation and molecular docking of curcumin analogues as antioxidant, cyclooxygenase inhibitory and anti-inflammatory agents. Bioorg Med Chem Lett. 2005;15:1793-7.

9. Koeberle A, Muñoz E, Appendino GB, Minassi A, Pace S, Rossi A, et al. SAR studies on curcumin's pro-inflammatory targets: discovery of prenylated pyrazolocurcuminoids as potent and selective novel inhibitors of 5-lipoxygenase. J Med Chem. 2014;57:5638-48.

10. Narumoto O, Matsuo Y, Sakaguchi M, Shoji S, Yamashita N, Schubert D, et al. Suppressive effects of a pyrazole derivative of curcumin on airway inflammation and remodeling. Exp Mol Pathol. 2012;93:18-25.

11. Arthur JS, Ley SC. Mitogen-activated protein kinases in innate immunity. Nat Rev Immunol. 2013;13:679-92.

12. Ben $\mathrm{P}$, Liu J, Lu C, Xu Y, Xin Y, Fu J, et al. Curcumin promotes degradation of inducible nitric oxide synthase and suppresses its enzyme activity in RAW 264.7 cells. Int Immunopharmacol. 2011;11:179-86.

13. Guimarães MR, Leite FR, Spolidorio LC, Kirkwood KL, Rossa C Jr. Curcumin abrogates LPS-induced pro-inflammatory cytokines in RAW 264.7 macrophages. Evidence for novel mechanisms involving SOCS-1, -3 and p38 MAPK. Arch Oral Biol. 2013;58:1309-11.

14. Yoon YD, Kang JS, Han SB, Park SK, Lee HS, Kang JS, et al. Activation of mitogen-activated protein kinases and AP-1 by polysaccharide isolated from the radix of Platycodon grandiflorum in RAW 264.7 cells. Int Immunopharmacol. 2004;4:1477-87.

15. Changtam C, Hongmanee P, Suksamrarn A. Isoxazole analogs with highly potent multi drug-resistant antimycobacterial activity. Eur J Med Chem. 2010;45:4446-57.

16. Griess JP. Determination of nitrite and nitrate by Griess reaction. Ber Deutsch Chem Ges. 1879;12:426-9.
17. Miyahara K, Murayama H, Wakabayashi H, Kurihara T, Hashimoto K, Satoh K, et al. Inhibition of LPS-stimulated NO production in mouse macrophage-like cells by benzocycloheptoxazines. Anticancer Res. 2008;28:2657-62.

18. He Y, Yue Y, Zheng X, Zhang K, Chen S, Du Z. Curcumin, inflammation, and chronic diseases: How are they linked? Molecules. 2015;20:9183-213.

19. Min KJ, Um HJ, Cho KH, Kwon TK. Curcumin inhibits oxLDL-induced CD36 expression and foam cell formation through the inhibition of p38 MAPK phosphorylation. Food Chem Toxicol. 2013;58:77-85.

20. Leong SW, Mohd Faudzi SM, Abas F, Mohd Aluwi MF, Rullah K, Lam KW, et al. Nitric oxide inhibitory activity and antioxidant evaluations of 2-benzoyl-6-benzylidenecyclohexanone analogs, a novel series of curcuminoid and diarylpentanoid derivatives. Bioorg Med Chem Lett. 2015;25:3330-7.

21. Ben $\mathrm{P}, \mathrm{Liu} \mathrm{J}, \mathrm{Lu} \mathrm{C}, \mathrm{Xu} \mathrm{Y}, \mathrm{Xin} \mathrm{Y}, \mathrm{Fu}$ J, et al. Curcumin promotes degradation of inducible nitric oxide synthase and suppresses its enzyme activity in RAW 264.7 cells. Int Immunopharmacol. 2011;11:179-86.

22. Tocharus J, Jamsuwan S, Tocharus C, Changtam C, Suksamrarn A. Curcuminoid analogs inhibit nitric oxide production from LPS-activated microglial cells. J Nat Med. 2012;66:400-5.

23. Sreejayan, Rao MN. Nitric oxide scavenging by curcuminoids. J Pharm Pharmacol. 1997;49:105-7.

24. Zhao F, Gong Y, Hu Y, Lu M, Wang J, Dong J, et al. Curcumin and its major metabolites inhibit the inflammatory response induced by lipopolysaccharide: translocation of nuclear factor- $\mathrm{\kappa B}$ as potential target. Mol Med Rep. 2015;11:3087-93.

25. Gupta SC, Prasad S, Kim JH, Patchva S, Webb LJ, Priyadarsini IK, et al. Multitargeting by curcumin as revealed by molecular interaction studies. Nat Prod Rep. 2011;28:1937-55.

26. Zhou Y, Zhang T, Wang X, Wei X, Chen Y, Guo L, et al. Curcumin modulates macrophage polarization through the inhibition of the toll-like receptor 4 expression and its signaling pathways. Cell Physiol Biochem. 2015;36:631-41.

27. Meng Z, Yan C, Deng Q, Gao DF, Niu XL. Curcumin inhibits LPS-induced inflammation in rat vascular smooth muscle cells in vitro via ROS-relative TLR4-MAPK/NF-kB pathways. Acta Pharmacol Sin. 2013;34:901-11.

28. Moreno-Fierros L, García-Hernández AL, Ilhuicatzi-Alvarado D, Rivera -Santiago L, Torres-Martínez M, Rubio-Infante N, et al. CrylAc protoxin from Bacillus thuringiensis promotes macrophage activation by upregulating CD80 and CD86 and by inducing IL-6, MCP-1 and TNF- $\alpha$ cytokines. Int Immunopharmacol. 2013;17:1051-66.

29. Feng D, Ling WH, Duan RD. Lycopene suppresses LPS-induced NO and IL-6 production by inhibiting the activation of ERK, p38MAPK, and NF-kappaB in macrophages. Inflamm Res. 2010;59:115-21.

30. Wang YJ, Pan MH, Cheng AL, Lin LI, Ho YS, Hsieh CY, et al. Stability of curcumin in buffer solutions and characterization of its degradation products. J Pharm Biomed Anal. 1997;15:1867-76. 